\title{
HIGH ANGULAR RESOLUTION BINARY STAR INTERFEROMETRY
}

Harold A. McAlister

Department of Physics, Georgia State University, Atlanta, Georgia, U.S.A.

\section{$\underline{\text { ABSTRACT }}$}

The benefits and limitations of the application of high angular resolution interferometry to the study of binary stars are considered. The principle advantage of such observations is that they provide data complementary to parameters determined by other techniques and permit the direct determination of masses, angular diameters and distances to systems which would otherwise be inaccessible to such complete analysis. These values then directly lead to linear diameters, absolute magnitudes, absolute emergent fluxes and effective temperatures. The prospects for significantly increasing our knowledge of these fundamental astrophysical parameters are extremely promising. The applications of interferometry to surveys for new binary stars, to star clusters, and to wider visual binaries with the goal of detecting planetary perturbations are also briefly considered.

\section{INTRODUCTION}

Binary star interferometry is by no means a new field in astronomy. The resolution of the spectroscopic binary Capella with the Michelson interferometer at Mt. Wilson ${ }^{1}$ was a spectacular and promising beginning more than half a century ago, at about the same time that Hertzsprung was first applying photography to the study of binary stars. The subsequent ups and downs of the field, which never could match the initial success in its brief intensity of excitement, are well documented by W. S. Finsen ${ }^{2}$ who concludes that the early overestimation of accuracy and underestimation of difficulty were in part responsible for the low level of activity. The most valuable contribution interferometry has made to binary star astronomy comes from Finsen's twenty years of observations of southern binary stars from Johannesburg with his highly compact eyepiece interferometer on the 26 -inch refractor. It is remarkable that with Finsen's clear demonstration of a successful program, no 
serious, longterm program was initiated in the northern hemisphere with its relatively greater supply of large telescopes. Systematic interferometer observations of binary stars ceased altogether with Finsen's retirement.

Additional modern contributions to binary star interferometry have been made by Kulagin ${ }^{3}$ who used a special periscopic Michelson interferometer to observe Capella more than 150 revolutions after the last observations by Merril1 ${ }^{4}$ in 1921. Kulagin essentially verified Merrill's determination of the orbital elements. An automated, photoelectric version of Anderson's modification of the Michelson interferometer was developed by Wickes and Dicke $^{5}$, but has thus far seen only limited application with telescopes not exceeding 60 inches in aperture. ${ }^{6-8}$ A new Michelson interferometer with a 1.87 meter fixed baseline has been constructed by the Royal Observatory Edinburgh to be used for binary star observations from that observatory's Italian station. ${ }^{9}$

Speckle interferometry, as first suggested by Labeyrie in 1970, has in recent years begun to produce a significant collection of binary star observations.10-16 Speckle observers to date have succeeded in resolving nearly 40 previously directly unresolved systems with angular separations down to 0.02 arcseconds. Continuing speckle programs, aimed primarily at observing binary stars, are currently underway with the 2.5-meter telescope at Herstmonceux ${ }^{13}$ and the 2.1-meter and 4-meter telescopes at Kitt Peak. 14

The very high angular resolution obtainable from multiple telescope interferometers has been most strikingly demonstrated by the success in resolving the 4.0 day spectroscopic binary $\alpha$ Virginis with the intensity interferometer at Narrabri ${ }^{17}$. The widespread application of such high angular resolution interferometry to binary star astronomy would initiate a new era for this classical field which has long been the sole direct source for one of the most fundamental of all astrophysical parameters - stellar mass. The angular resolutions obtainable by interferometers discussed during this Colloquium could finally put aside the obstacle of non-overlapping selection effects that have prevented binary star astronomers from vastly increasing their contribution to the collection of fundamental astrophysical data. The oft lamented inability of the various techniques to completely 
determine all the parameters of a binary system and the inaccessibility of al1 but a few systems to more than one observational method are well known. Indeed, it is this circumstance which preserves the classification scheme which, as Batten ${ }^{18}$ points out, is useful for very little besides classifying binary star astronomers.

Interferometric observations provide the same set of data as do visual observations, 1.e. position angle, angular separation and, hopefully, magnitude difference, but with an orders of magnitude gain in limiting angular resolution, interferometry can bring spectroscopic and eclipsing binaries into the realm of the "visual" observer By means of complementary observations, various parameters can then be unraveled which would be otherwise lost to observational selection.

Various potential applications of interferometry to binary star astronomy are summarized in succeeding sections. It is not generally necessary to distinguish between the various kinds of modern interferometry, and the emphasis here will be on the combination of interferometry with other techniques. Although interferometry can clearly be extremely useful in discovering new binary and multiple stars, detailed studies of such systems by interferometry alone will be limited by the same restrictions imposed on visual orbit determinations, i.e. need for an accurate trigonometric parallax to obtain a meaningful mass sum from Newton's form of Kepler's Third Law and the need for a spectroscopically or astrometrically determined mass ratio. A tabular summary of the parameters determinable by the various techniques is given by Batten $^{18}$ The clearcut nature of the applications described below, and summarized in Table 1 , serves to emphasize their fundamental importance as well as the great difficulties which have so far permitted only limited successes.

\section{OBSERVATION OF SPECTROSCOPIC BINARIES}

The gap between the visual and spectroscopic binaries is the most pronounced of the non-overlapping selection effects in binary star astronomy Those few systems which can be observed by both techniques are generally at the limits of sensitivity for the two methods in terms of very small angular 
TABLE 1. Combined Studies of Binary Stars

Double-1ined Spectroscopic Binaries:

$\begin{array}{ccc}\text { Interferometry } & + \text { Spectroscopy } & \text { Results } \\ \text { p's } & \mathrm{P}, \mathrm{T}, \omega, \mathrm{e}, \mathrm{k}_{1}, 2 & \mathrm{i}, \mathrm{M}_{1}, \mathrm{M}_{2}, \pi\left(\mathrm{L}_{1}, \mathrm{~L}_{2}\right) \\ \theta^{\prime} \mathrm{s} & \mathrm{P}, \mathrm{T}, \omega, \mathrm{e}, \mathrm{k}_{1}, 2 & \mathrm{i}, \mathrm{M}_{1}, \mathrm{M}_{2}, \Omega \\ \text { i,a" } & \left(\mathrm{M}_{1}, \mathrm{M}_{2}\right) \mathrm{Sin}^{3}{ }^{\mathrm{i}, \text { aSini }} & \mathrm{M}_{1}, \mathrm{M}_{2}, \pi\left(\mathrm{L}_{1}, \mathrm{~L}_{2}\right)\end{array}$

Single-lined Spectroscopic Binaries:

\begin{tabular}{|c|c|c|c|c|c|}
\hline Interferometry & + & Spectroscopy & + & Other & Results \\
\hline$\theta^{\prime} s$ & & $P, T, e, \omega, k$ & & - & $i, M_{2}^{3} /\left(M_{1}+M_{2}\right)^{2}, \Omega$ \\
\hline$i, a^{\prime \prime}, \Delta \mathrm{m}$ & & $f(M)$ & & $\operatorname{trig} \pi$ & $\mathrm{M}_{1}, \mathrm{M}_{2}, \mathrm{~L}_{1}, \mathrm{~L}_{2}$ \\
\hline$i, a^{\prime \prime}, \Delta \mathrm{m}$ & & $f(M)$ & & M-L re1 & $\mathrm{M}_{1}, \mathrm{M}_{2}, \pi\left(\mathrm{L}_{1}, \mathrm{~L}_{2}\right)$ \\
\hline
\end{tabular}

Astrometric Binaries:

Interferometry

$\mathrm{a}^{\prime \prime}, \Delta \mathrm{m}$

$+\quad$ Astrometry

Resu1ts

$\alpha^{\prime \prime}, \pi$

$\mathrm{M}_{1}, \mathrm{M}_{2}, \mathrm{~L}_{1}, \mathrm{~L}_{2}$

Eclipsing Binaries:

$\begin{array}{ccccc}\text { Interferometry } & +\quad \text { Photometry } & + & \text { Other } & \text { Results } \\ a^{\prime \prime} & \mathrm{r}_{1}, \mathrm{r}_{2}, \mathrm{i}, \Delta \mathrm{m} & \mathrm{DSB} & \pi\left(\mathrm{L}_{1}, \mathrm{~L}_{2}\right), \mathrm{M}_{1}, \mathrm{M}_{2}, \mathrm{R}_{1}, \mathrm{R}_{2}\end{array}$


separation and/or very small velocity amplitude so that the combined orbital solution lacks the desired accuracy. Spectroscopic binaries are favored by the selection effects of short period and high orbital inclination, essentially independent of distance (so long as the objects are bright enough to obtain measurable spectra) It has been shown ${ }^{19}$ that for a given period $P$ in days and parallax $\pi$, one would expect to find an approximate average separation within a large sample of binary stars of

$$
\langle\rho\rangle=\left(1.16 \times 10^{-2}\right) \pi \mathrm{P}^{2 / 3} \text { arcseconds. }
$$

Thus an instrument with the capability of resolving systems with separations as small as $10^{-4}$ arcseconds should be able to measure binaries within 25 parsecs with periods as short as 2.4 hours (a rather meaningless extrapolation) or binaries within 1000 parsecs having periods longer than 25 days. Reducing the limiting separation to $10^{-3}$ arcseconds leaves a minimum average period for stars resolvable within 25 parsecs of approximately 3.2 days.

TABLE 2. Minimum Separations for Nearby Spectroscopic Binaries

\begin{tabular}{|c|c|c|c|}
\hline $\log \rho_{\min }$ & $\begin{array}{l}\text { required } \\
\text { resolution }\end{array}$ & $\begin{array}{c}\text { number of } \\
\text { systems }\end{array}$ & $\begin{array}{l}\text { minimum } \\
\text { baseline }\end{array}$ \\
\hline$<-3.50$ & - & 1 & - \\
\hline-3.50 to -3.01 & $0: 0003$ & 7 & $155 \mathrm{~m}$ \\
\hline-3.00 to -2.51 & 0.0010 & 10 & 46 \\
\hline-2.50 to -2.01 & 0.0032 & 11 & 15 \\
\hline-2.00 to -1.51 & 0.010 & 5 & 5 \\
\hline-1.50 to -1.01 & 0.032 & 4 & 1.5 \\
\hline$\geq-1.00$ & $0: 10$ & 17 & - \\
\hline
\end{tabular}

The "Catalogue of Stars Within 25 Parsecs"20 contains 55 stars which are common to "The Sixth Catalogue of the Orbital Elements of Spectroscopic Binary Stars" 21 Several of these systems are also visual binaries and ten are double-1ined. The relatively accurately known trigonometric parallaxes and the spectroscopic elements can be used to determine a minimum angular separation at nodal passage which is unaffected by projection effects arising 
from the unknown inclination. 19 Table 2 contains the distribution of minimum angular separations for nearby spectroscopic binaries along with the baseline required for resolution at $450 \mathrm{~nm}$ wavelength. The shortest period spectroscopic binary contributing to Table 2 is 1 Boo with a period of 0.268 day and the closest minimum angular separation is 0.00030 for $\alpha$ Tri. It is fascinating to note that all of the separations in Table 2 are within reach of the Narrabri intensity interferometer ${ }^{22}$; unfortunately, angular separation is not the only consideration for successful results. Of the nearby spectroscopic binaries, four have primaries which are classified as giant stars and another nine are subgiants. Main sequence stars earlier than spectral type Fo number only eight with no systems having components earlier than AO. Only five of the 55 systems have combined $V$ magnitudes fainter than +7.0 .

If one chooses to consider stars with distances exceeding 25 parsecs and thus decreasing the certainty of the predicted minimum separation, then the diversity of candidates for resolution increases dramatically. In a previous study aimed primarily at collecting a sample of stars suitable for resolution by speckle interferometry ${ }^{19}$, spectroscopic binaries were sought in the catalogue of Batten ${ }^{21}$ with predicted values of (a Sin i)" > 0!005. The compilation resulted in 73 systems that might be resolvable by speckle interferometry $\left(\rho_{\text {min }} \geq 0: 02\right)$. Thirteen of these objects have so far been resolved by all speckle observers; however, a few of the newly resolved secondaries may turn out to be previously unknown companions. The larger source list contained 112 objects with spectral types ranging from 09 to M2. The sample includes 39 systems with at least one giant companion with spectral types ranging from 09 to M1. There are 13 systems containing supergiants with predicted angular semi-major axes exceeding 0".005.

The resolution of a significant sample of short and long period spectroscopic binaries of early spectral type may reveal differences in the massluminosity relations for stars which are possibly interacting with their companions as compared to wider systems where the components are essentially physically isolated. In order to increase the astrophysical return from interferometry, it is highly desirable to perfect ways of reliably measuring magnitude and color differences within resolved systems. 
Clearly, the possibilities for dramatically increasing our knowledge of the empirical mass-luminosity relation are seemingly unlimited if a highly sensitive, long baseline interferometer can successfully be built. Even with a limiting magnitude restricting study to only the naked eye stars, such an instrument could have a profound effect upon fundamental astrophysics.

\subsection{Double-Lined Systems}

Observations of double-lined spectroscopic binaries offer the most promising returns from high angular resolution binary star interferometry= It is, first of all, these objects with small magnitude differences that are most likely to be resolved. Secondly, because the line of sight components of motion can be measured for both members, the mass ratio and linear value of a Sin $i$ for the relative orbit are known. The great usefulness of these objects derives from the combination of the angular measures from the "visual" observations with the linear measures of the spectroscopic observations to determine the distance to the system and the masses of the individual components. None of these parameters can be derived from either set of observations alone.

There are a variety of ways in which the interferometric and spectroscopic results can be combined. For example, the visual orbit derived from the interferometry alone yields an inclination $i$ and semi-major axis $a "$ which can be applied to the spectroscopically determined values of $M_{1} \operatorname{Sin}^{3} i, M_{2} \operatorname{Sin}^{3} i$ and a Sin $i$ to determine distance (and thus luminosities if $\Delta \mathrm{m}$ is available) and masses. Alternately, the spectroscopic elements can be adopted and the interferometric observations can be applied in sets of measured separations and position angles separately to determine the unknown parameter pairs $(\pi, i)$ and $(i, \Omega)$ respectively. This has been done for the double-1ined system 12 Persei from speckle observations with the 4-meter telescope at Kitt Peak. ${ }^{3}$ The six measured separations yielded $1=123: 5$ while the position angles gave $i=120: 6$ when the spectroscopic elements $\mathrm{P}, \mathrm{T}, \mathrm{e}, \omega$ and a Sin $i$ were adopted. These independently determined values of $i$ are in reasonably close agreement. A similar procedure was carried out for Capella by Anderson ${ }^{1}$, later by Merril1 ${ }^{4}$ and most recent $1 y$ by Finsen ${ }^{24}$ who included the modern interferometer observa- 
tions of Kulagin. ${ }^{3}$ The analysis of the 4.0 day spectroscopic system comprising $\alpha$ Virginis from observations with the intensity interferometer ${ }^{17}$ was done after adopting a set of spectroscopic elements and revealed an angular semimajor axis of only $0.00154 \pm 0.00005$ arcseconds. In this last analysis, the unknown elements were determined by adjusting trial elements in an expected normalized correlation function and iteratively fitting to the observed correlations without the intermediate step of explicitly determining the angular separation and position angles.

The Narrabri observations of $\alpha$ Virginis also produced the angular diameter of the B1.5 IV-V primary and thus directly lead to the absolute flux at the stellar surface and the effective temperature. The results for $\alpha$ Virginis are all consistent with the empirical mass-luminosity and mass-radius relations and serve as a model of completeness for future combined interferometric, spectroscopic and photometric studies. Preliminary determinations of the angular diameters of the components of Capella have also been made with the amplitude interferometer at CERGA. 25 Their values are about $30 \%$ smaller than values expected for blackbodies with the effective temperatures and distance of Capella.

Methods of orbit solution have been developed which permit the direct combination of observations of separation and position angle with radial velocities so that the derived elements have contributions from all possible observations.26,27 These techniques have the advantages of supplying formal, statistical error estimates and are most useful in dealing with data samples of uniform quality. An interesting test of these and similar techniques would be a combined solution incorporating the rather large collection of interferometric and spectroscopic data now available for Capella.

The principal outstanding virtue of combining interferometric and doublelined spectroscopic observations is the resulting direct determination of the distance to the system. Distances determined in this manner are independent of the effects of interstellar extinction and can potentially penetrate to distances well beyond the effective accurate limit for trigonometric parallaxes while still preserving the directness and uniqueness of a simple geometric technique. 


\subsection{Single-Lined Systems}

of course, the majority of spectroscopic binaries are single-lined and do not so easily yield a complete set of orbital and physical parameters. The principal difficulty here is the incomplete knowledge of the linear scale of the relative orbit, thus prohibiting direct geometric determinations of the distance. A determination of the relative visual orbit from the interferometer observations alone gives the orbital inclination and the spectroscopically determined mass function simplifies to just $M_{2}^{3} /\left(M_{1}+M_{2}\right)^{2}$. If the parallax is already known, then Kepler's Third Law gives the mass sum, and the individual masses are then readily available for inclusion in the mass-1uminosity relation.

If the parallax is unknown, as will be likely in most cases, then one may use the mass-1uminosity relation to find the individual masses and a dynamical parallax if the magnitude difference can be measured with reasonable accuracy. The mass-luminosity relation leads to a mass ratio of

$$
\frac{M_{2}}{M_{1}}=10^{-\kappa \Delta m}
$$

where $-k$ is the slope of the mass-luminosity relation and is a function of the particular magnitude system employed. The slope of the mass-luminosity relation is also known to increase rather abruptly for stars brighter than $M_{b}=+7.5$, but the spectroscopy can probably decide which value of $k$ is appropriate. Assuming a representative value of $k=0.10 \pm 0.02^{28}$ and hoping that $\Delta \mathrm{m}$ can be measured to within \pm 0.2 magnitudes, then the mass ratio is given to within $\pm 15 \%$ for $\Delta \mathrm{m}=3.0$. The accuracy of the mass ratio increases to $\pm 5 \%$ for $\Delta \mathrm{m}=0.0$. This mass ratio can then be applied to the simplified mass function $M_{2}^{3} /\left(M_{1}+M_{2}\right)^{2}$ to give the individual masses and then, by means of Kepler's Third Law and the interferometric angular semi-major axis, the paral1ax. This method only works here because the $\operatorname{Sin}^{3} i$ factor has been eliminated from the mass function by the interferometric determination of the visual orbit. This represents a significant gain in the potential from single-lined spectroscopic binary mass functions which can otherwise be used only in a 
statistical sense.

There may also be individual cases for which the masses can be directly determined. For example, the primary component of the 9.2 year single-1ined spectroscopic system $\eta$ Orionis is also an 8 day double-lined eclipsing system in which $\operatorname{Sin}^{3} i$ must be near unity. Since the individual masses $M_{1}$ and $M_{2}$ comprising the short period system can be determined, then the value of $\mathrm{M}_{3}$ for the long period component can be estimated if one makes the assumption of coplanarity between the long and short period systems. Fortunately in this case, the long period system has been resolved by speckle interferometry at a separation of approximately 0.05 arcseconds $^{29}$, and the inclination can eventually be directly determined. The inftial observations do hint toward a high inclination. If the assumption of coplanarity is adopted temporarily for $\eta$ Orionis, then the masses and luminosities can be determined for the three early B spectral type components which are found to be about 400 parsecs from the sun. Here, again, the distance is determined from purely geometric principles.

It is also to be hoped that special efforts will be made to spectroscopically detect the faint secondaries in single-lined systems by employing modern low-noise detectors in the red end of the spectrum. The recent detection of Algo1 $B^{30}$ is an exciting example of this. Not only have the masses $M_{A}$ and $M_{B}$ been determined, but the B component has been shown to fill its Roche lobe and thus transporting mass to the $A$ component resulting in the previously observed radio and $x$-ray emission. The distance to the system and the mass of the spectroscopic/speckle interferometric/astrometric component Algol C should be accurately determined by continued interferometric observations. Detection of the third components for several triple systems has also recently been reported. And, of course, interferometer observers should also observe at wavelengths likely to decrease the magnitude difference in a single-lined binary

Thus there are numerous ways in which the interferometric resolution of single-1ined spectroscopic systems can directly lead to fundamental astrophysical parameters. 


\section{OBSERVATIONS OF ASTROMETRIC AND ECLIPSING BINARIES}

Direct resolution of an astrometric binary, with a photocentric semimajor axis $\alpha^{\prime \prime}$, along with an accurate determination of its magnitude difference immediately gives the individual masses and luminosities whose accuracies are strongly influenced by the accuracy of the trigonometric parallax. A detailed discussion of the current status of unresolved astrometric binaries is given by van de Kamp. ${ }^{31}$ The large expected magnitude differences and the apparent faintness of the invisible secondaries for these objects will make them extremely challenging for interferometer observers and there will be clear advantages of observing them in the infra-red. Successful mass determinations for astrometric binaries would shed considerable light on the low mass end of the mass-luminosity relation and provide insight to the transition from stellar to planetary masses. It remains highly unlikely, however, that any of the suspected planetary mass companions can be directly resolved with present day technology. We shall return to the possibility of detecting extra-solar planets in Section 4.2.

The determination of the angular semi-major axis for an eclipsing binary which is also a double-lined spectroscopic binary is sufficient to yield the parallax of the system (and thus the luminosities of the components), the Individual masses and the absolute individual stellar radii. This is done without reference to a luminosity-effective temperature relation since the parallax of the system can be directly determined by the addition of the interferometer observations, and would provide a valuable check on the traditional technique of placing eclipsing/spectroscopic binaries on the massluminosity relation.

\section{OTHER APPLICATIONS OF BINARY STAR INTERFEROMETRY}

Although binary star interferometry will undoubtedly be most productive in the area of providing new fundamental astrophysical data in the form of stellar masses, distances and luminosities, it will certainly play a role in other areas currently challenging binary star astronomy. These areas include the frequency of incidence of binary and multiple star systems and the implications of binary stars to the existence of planetary systems. We shall now 
briefly consider these and other applications.

\subsection{Surveys for New Binary Stars}

Surveys among restricted samples of stars would be worthwhile undertakings for interferometer observers as long as the special selection criteria which favor interferometry above other techniques are kept in mind. In recent years observations have shown that the number of binary and multiple stars in our galaxy is surprisingly high, perhaps approaching 100 percent for solar type stars. 32 The frequency of non-single stars has important bearing on stellar origin and evolution, and the determination of binary and multiple star frequency is a major goal of modern binary star astronomy

Because of the above mentioned selection criteria, it seems that interferometry would be uniquely suited to discovering binary stars with periods on the order of hundreds of days that would otherwise be too close for visual observers and have undetectible velocity amplitudes for spectroscopists. This intermediate range of sensitivity coupled with the hoped for ability to detect magnitude differences as large as five magnitudes would allow interferometry to play a valuable role in searching for new binary stars.

Speckle interferometry with the largest available telescopes is already capable of taking on this task within the limitations of available telescope time and the apparent current sensitivity to magnitude differences less than about 2.5 magnitudes. It is interesting to note that a number of previously unknown binaries or components have been found by speckle interferometry in a mostly serendipitous fashion while attempting to resolve known spectroscopic pairs. Some of these have already shown considerable orbital motion. It is unlikely that the very high angular resolution of a long baseline interferometer would offer significant and unique gains in surveys for new binary stars since the objects such an interferometer could ideally observe are also very suited to spectroscopy. It would also seem that the very large number of spectroscopic binaries resolvable by a long baseline interferometer could more than tie-up all the time available for binary star astronomy on the instrument.

Surveys that are undertaken should be directed at astrophysically interesting groups of stars such as certain types of variable stars, novae, blue 
stragglers, etc. The sample of nearby stars should be observed for previously unknown rapidly moving pairs with large magnitude differences in order to more completely define the low mass end of the mass-luminosity relation. To further increase the uniqueness of interferometer surveys, observations in the infrared should be made to detect cool, low mass components that might be overlooked in a spectroscopic survey due to the resulting low velocity amplitudes of the primary components. New binary stars discovered in the Hyades cluster could make a significant contribution toward recent efforts to accurately determine the distances and mass-1uminosity relation for that fundamentally important cluster. And, of course, interferometer observations should be obtained for objects with indications of duplicity from lunar occultation observations, suspected variable radial velocity, excess luminosity, etc. Interferometry will undoubtedly lead to a number of newly resolved bright binary stars which not only will call for continuous interferometric observation, but will require the complementary observations of spectroscopists and photometrists before the full astrophysical potential from these systems can be realized.

\subsection{Detection of Planetary Perturbations in Binary Systems}

A great deal of interest currently exists in the detection of extra-solar planetary systems and the related question of the frequency of life in the universe. Although large magnitude differences would make the direct detection of a Jovian-like planet about a nearby star unlikely, interferometry, by virtue of the high accuracy with which observations of binary stars can be made, can contribute to the search by looking for very small perturbations in the orbital motion of moderately wide binary stars. Speckle interferometry has been suggested as a technique for continued observation of visual binaries with separations out to a few arcseconds. 33

It is important to note that this group of objects is not amenable to study by the traditional techniques due to blending effects from the close images. The existence of stable planetary orbits with conditions supportive of life in binary star systems has long been questioned. However, Harrington ${ }^{34}$ has recently shown, by means of numerical integrations of the general three body problem, that stable and habitable planets can exist within binary star 
systems. Thus any significant search for extrasolar planetary systems should not exclude binary stars on the grounds of either low probability of existence of planets or the inability of the traditional techniques to handle these objects.

\subsection{Observations of Star Clusters}

The relative positions of cluster members can be determined by interferometric observations in a procedure employing the measurement of many pairs of stars. For example, speckle interferometry with a sufficiently sensitive speckle camera is ideally suited to the study of the central regions of globular clusters (as projected onto the sky) where the number density of stars 35 is such to insure that numerous overlapping pair configurations can be established with separations less than the atmospheric isoplanatic patch size. Through measurements of such overlapping pairs, one can work through the central cluster region in a variety of closed paths and establish a very tight positional framework with relative positions accurate to within \pm 0.001 arcsecond. With a time baseline of 10 years, relative annual proper motions could be determined with accuracies approaching \pm 0.0001 . These would then be extremely useful in studying cluster dynamics.

\section{CONCLUSION}

High angular resolution interferometry clearly presents exciting possibi11ties for significantly increasing the return from an investment of not inconsiderable effort in binary star astronomy: Binary stars should be a primary observational goal and, indeed, a raison d'etre for a long baseline interferometer which should be designed with the requirements for successfully observing these objects in mind. Smaller amplitude interferometers and speckle interferometry will hopefully continue to produce useful results, and the evolution of binary star astronomy and its contribution to modern astrophysics will be firmly maintained. 


\section{REFERENCES}

1) J.A. Anderson, Astrophys. Journ., 51, 263, 1920.

2) W.S. Finsen, Astrophys. Space Sci., 11, 13, 1971.

3) E.S. Kulagin, Soviet Astron., 14, 445, 1970.

4) P.W. Merril1, Astrophys. Journ., 56, 40, 1922.

5) W.C. Wickes and R.H. Dicke, Astron. Journ., 73, 757, 1973.

6) W.C. Wickes and R.H. Dicke, Astron. Journ., 79, 1433, 1974.

7) W.C. Wickes, Astron. Journ., 80, 655, 1975.

8) W.C. Wickes, Astron. Journ., 80, 1059, 1975.

9) R.Q. Twiss and W.J Tango, Rev. Mexicana Astron. Astrof., 3, 35, 1977

10) D.Y Gezari, A. Labeyrie and R.V. Stachnik, Astrophys. Journ. (Letters), 173, L1, 1972 .

11) A. Labeyrie, D. Bonneau, R.V Stachnik and D.Y Gezari, Astrophys. Journ. (Letters), 194, L147, 1974.

12) A. Blazit, D. Bonneau, L. Koechlin and A. Labeyrie, Astrophys. Journ. (Letters), 214, L79, 1977

13) B.L. Morgan, D.R. Beddoes, R.J. Scaddan and J:C. Dainty, Mon. Not. R. Astron. Soc., 183, 701, 1978.

14) H.A. McAlister, Astrophys. Journ., 215, 159, 1977

15) H.A. McAlister, Astrophys. Journ., in press.

16) H.A. McAlister and K.A. DeGioia, Astrophys. Journ., in press.

17) D. Herbison-Evans, R. Hanbury Brown, J Davis and L.R. Allen, Mon. Not. R. Astron. Soc., 151, 161, 1971.

18) A.H. Batten, "Binary and Multiple Systems of Stars" (Pergamon Press, New York), 1973.

19) H.A. McAlister, Pub. Astron. Soc. Pac., 88, 317, 1976.

20) R. Woolley, E.A. Epps, M.J. Penston and S.B. Pocock, Royal Obs Ann. No. 5, 1970.

21) A.H. Batten, Pub. Dominion Astrophys. Obs., 13, No. 8, 1967

22) J. Davis, Proc. Astron. Soc. Australia, 3, 26, 1976.

23) H.A. McAlister, Astrophys. Journ., 223, 526, 1978.

24) W.S. Finsen, IAU Comm. 26 Circ. d'Inf., No. 66, 1975.

25) A. Blazit, D. Bonneau, M.Josse, L. Koechlin, A. Labeyrie and J.L. Oneto, Astrophys. Journ. (Letters), 217, L55, 1977

26) C.L. Morbey, Pub. Astron. Soc. Pac., 87, 689, 1975.

27) D.G. Monet, Astrophys. Journ. (Letters), 214, L133, 1977 
28) D.L. Harris, K.Aa. Strand and C.E. Worley, "Basic Astronomical Data", ed. K.Aa. Strand (Univ. Chicago Press, Chicago), p. 273, 1963.

29) H.A. McAlister, Pub. Astron. Soc. Pac., 88, 957, 1976.

30) J. Tomkin and D.L. Lambert, Astrophys. Journ. (Letters), 222, L119, 1978.

31) P van de Kamp, Ann. Rev. Astron. Astrophys., 13, 295, 1975.

32) H.A. Abt and S.G. Levy, Astrophys. Journ. Supp., 30, 273, 1976.

33) H.A. McAlister, Icarus, 30, 789, 1977.

34) R.S. Harrington, Astron. Journ., 82, 753, 1977.

35) C.J. Peterson, Astron. Journ., 81, 617, 1976.

\section{DISCUSSION}

M.L. Aizenman: What is the status of interferometric measurements on binary systems that appear to have compact objects?

H.A. McAlister: These objects are found in systems with periods too short to be resolved by classical speckle interferometry with a single aperture and have thus not yet been successfully resolved. They might be resolved by a long baseline interferometer in the future.

M. Shao: What is the effect of the magnitude limit on the feasibility of interferometric measurements of globular cluster proper motions?

H.A. McAlister: The current state of the art using film speckle cameras is 8 th-9th magnitude, but the need is for 15 th magnitude which should be achievable in the future. Thirteenth magnitude has been achieved by Labeyrie and his collaborators and, I understand, by the speckle group at the Steward Observatory. 